\title{
La representación de la heroína ordinaria Evangelina en los medios de comunicación
}

\author{
Sascha Maria Frisdahl
}

Independiente

saschafrisdahl@gmail.com

RESUMEN

La siguiente investigación utiliza el método del análisis crítico del discurso de Norman Fairclough para analizar la representación de Evangelina Chamorro, quien sobrevivió a un huaico del año 2017 en el Perú. El objetivo del estudio es analizar cómo se representa a una heroina ordinaria en los medios de comunicación en el Perú a partir de artículos de dos periódicos nacionales: Trome y La República. El análisis se realiza a partir de una muestra de seis artículos - tres de Trome y tres de La República一, recogidos entre el 16 de marzo de 2017 y el 13 de mayo de 2017.

El estudio se ubica dentro un marco teórico del heroísmo ordinario y la representación del género femenino en los medios de comunicación. La investigación concluye que los cuatro discursos principales en la representación de Evangelina son: sobreviviente, mujer, religiosa y pobre. Ambos periódicos crean una imagen heroica de Evangelina utilizando valores tradicionales del género en sociedades latinoamericanas. La identidad social que predomina en la representación de Evangelina es la de madre. Es decir, esta gira alrededor de la maternidad, pero el análisis también concluye que no es fija, ya que cambia según el contexto.

Palabras clave: análisis crítico del discurso, héroes ordinarios, género, Evangelina Chamorro, medios de comunicación, noticieros. 


\section{The Representation of the Ordinary Heroine Evangelina In the Mass Media}

\section{ABSTRACT}

The following study uses the critical discourse analysis by Norman Fairclough as a method to analyze the representation of Evangelina Chamorro, who survived a mudslide in Peru in 2017. The purpose of this study is to investigate how the mass media represents an ordinary heroine in Peru by analyzing articles from the two national newspapers: Trome and La República. This analysis is performed by drawing a sample of six articles - three from Trome and three from La Republica-, which have been collected between the 16th of March 2017 and the 13th of May 2017.

The theoretical framework of the study is ordinary heroism and the representation of women in the mass media. The study concludes that the four principal discourses in the representation of Evangelina is: survivor, woman, religious and poor. Both newspapers have the ability to create a heroic image around Evangelina using traditional values of gender of Latin American societies. Evangelina's social identity is predominantly presented through her role as a mother, but the analysis further concludes that her identity as a mother is not fixed since it changes depending on the context.

Keywords: critical discourse analysis, ordinary heroes, gender, Evangelina Chamorro, mass media, news. 


\section{INTRODUCCIÓN}

Evangelina Chamorro se hizo famosa cuando su lucha por sobrevivir a un huaico fue grabada con un celular y compartida en las redes sociales. Ella logró salir sola, sin ser rescatada por las personas que estaban observándola. Su lucha fue individual y solitaria. Ella pertenece a un grupo social poco representado en los medios de comunicación hoy en el Perú: es mujer, emigrante de la selva y con bajos recursos económicos. A pesar de ello, se ha convertido en la imagen de una heroína nacional. La construcción de héroes nacionales durante una crisis es un fenómeno usual. Es una construcción que requiere la participación de varios actores sociales, como la prensa, la Iglesia, instituciones educativas y la producción cultural. La construcción de un héroe nacional es producto de la sociedad y de su historia (McEvoy, 2008, p. 83).

El estudio visibiliza la ideología de género y critica a la heroína ordinaria que reproduce ciertos discursos de género, religión y pobreza. Es decir, que la imagen de Evangelina sustituye ciertos valores tradicionales que los ciudadanos deben seguir. Por lo tanto, la pregunta de la investigación es: ¿cómo se representa a Evangelina Chamorro en los medios de comunicación?

El estudio se ubica dentro del constructivismo social crítico, que es una crítica ideológica, lo que implica cuestionar las condiciones sociales injustas (Phillips y Jørgensen, 2002, pp. 176-179). La crítica de ideología tiene sus raíces en la teoría marxista, en la que las relaciones de poder en la sociedad son acompañadas por el lenguaje hegemónico que sistemáticamente encubre la realidad (Phillips y Jørgensen, 2002). Por lo tanto, intento visibilizar las relaciones de poder revelando las ideologías que hay detrás (Phillips y Jørgensen, 2002). Muchos de los sistemas de significado están tan establecidos que naturalizan nuestra percepción del mundo. Por consiguiente, el análisis crítico sirve para visibilizar estos sistemas de significado y generar discusiones, críticas y cambios. 
El análisis se ha realizado mediante una selección de artículos de dos periódicos limeños: La República y Trome. Ambos periódicos tienen distintas audiencias objetivas. Según un estudio realizado por la Compañía Peruana de Estudios de Mercado y Opinión Pública S.A.C., La República cuenta con mayor acogida dentro del nivel socioeconómico considerado medio-alto y alcanza un promedio de 110000 lectores por día dentro de Lima Metropolitana. Por su parte, Trome es el periódico más consumido en el país, tiene un promedio de 1923000 lectores por día en Lima Metropolitana y sus consumidores pertenecen al nivel bajo/ superior (Compañía Peruana de Estudios de Mercado y Opinión Pública S.A.C., 2017). Por un lado, se encuentra Trome, con un alcance masivo y popular. Por otro, La República, con menor número de lectores y cuya audiencia pertenece a sectores más acomodados.

La audiencia objetiva refleja la expresión periodística en la que Trome es considerado un diario popular o conocido con el calificativo de "diario chicha». Se caracteriza por tener artículos cortos y visuales; es decir, muestra un alto número de fotografías y videos, emplea colores llamativos, tiene un diseño directo y un acceso de información sobre entretenimiento, espectáculos y policiales (Muñoz, 2016, p. 99). Trome es un diario que ha sido criticado por sexualizar, victimizar y estereotipar a la mujer. La República, por lo contrario, presenta artículos largos y reflexivos. Asimismo, sus textos intentan tener un enfoque más explicativo y explorar a profundidad el contexto social.

Los artículos en los que se menciona a Evangelina Chamorro han sido recogidos entre el 15 de marzo y el 31 de mayo de 2017. La muestra para el análisis se basa en seis publicaciones: tres de Trome y tres de La República. Estas representan diferentes enfoques: el tema de Evangelina saliendo del huaico, la visita de la ministra de Salud, la primera entrevista con Evangelina y, finalmente, la madre.

El estudio se ubica dentro del constructivismo social crítico, que consiste en una investigación con una crítica ideológica (Phillips y Jørgensen, 2002, p. 176). En otras palabras, cuestionar las condiciones sociales injustas (p. 179). En este sentido, intento visibilizar el rol de los noticieros en constituir y reproducir identidades sociales y representar la información como objetiva y verdadera. La investigación es un intento por visibilizar las representaciones de género mediante un análisis de la representación de la heroína ordinaria que reproduce ciertos discursos de género relacionados con la religión y la pobreza. Muchos de los sistemas de significado están tan establecidos que naturalizan nuestra percepción del mundo (Phillips y Jørgensen, p. 178). El análisis crítico sirve, entonces, para visibilizar estos sistemas de significado y generar discusiones, críticas y cambios. 
El método utilizado es el análisis crítico del discurso, que se caracteriza por ser un método cualitativo de fenómenos sociales. Este método es útil para estudiar relaciones entre discursos y el desarrollo cultural (p. 60). El análisis crítico de Fairclough es útil para analizar el lenguaje como constituido por su función en la estructura social y el lenguaje que se utiliza dependiendo de su posición en el sistema social (Fairclough, 1992, p. 26). El significado de una palabra depende de la práctica social. Las palabras utilizadas no son decisión del individuo puro, son «socially variable and socially contested, and facets of wider social and cultural processes» (p. 185). El modelo tridimensional de Fairclough es utilizado para analizar los niveles textual y social, e incluir un análisis del medio. El medio, los artículos periodísticos, producen conocimiento utilizando una práctica discursiva que decide cómo se representa el conocimiento y cómo se produce.

A continuación identificaré el marco teórico del siguiente estudio, el cual se ubica principalmente dentro de dos áreas: el heroísmo ordinario y la representación del género femenino.

\section{LA REPRESENTACIÓN DEL HÉROE ORDINARIO EN LOS MEDIOS DE COMUNICACIÓN}

Evangelina expresa la necesidad de tener un héroe durante tiempos de crisis y representa ciertas normas y valores que funcionan como una guía para la sociedad. Según el investigador de culturas religiosas István Povedák (2014), los héroes son el resultado de una necesidad de la masa y expresan las necesidades socioculturales. Es un deseo creado desde abajo que funciona como una guía (p. 10). Evangelina surge de un efecto desde abajo; es decir, los medios se apropiaron del video que apareció en Facebook. El video es realizado con el celular de una persona que podría ser cualquier ciudadano que por coincidencia estuvo grabando cuando Evangelina salió del huaico. Este se hizo famoso rápidamente y los medios de comunicación empezaron a narrar la historia de Evangelina.

Evangelina no es un personaje que normalmente salga en los medios de comunicación en el Perú. Es una persona humilde, mujer y madre, migrante de la selva, pero que por su acción extraordinaria de sobrevivir al huaico sale en las noticias. Los artículos la describen como una mujer extraordinaria y luchadora, la vuelven un ejemplo a seguir para los demás. Es decir, su imagen constituye cierto modelo de cómo se debe ser y comportarse en la sociedad. Según el historiador Simon Wendt, los héroes ordinarios tienen normas, valores y creencias de ciertos 
grupos sociales. Además, tienden a ser modelos a seguir. Son símbolos de las normas e identidades dominantes, las cuales constituyen fuentes de autoridad y legitiman jerarquías sociales, políticas, culturales y raciales (Wendt, 2016, p. 10).

Una de las definiciones del héroe ordinario es que se reconoce por ser extraordinario; es decir, una persona común y corriente que hace algo notable. Según Oliva, Pérez-Latorre y Besalú (2015), se construye la imagen de la estrella sobre la dicotomía entre extraordinario y ordinario. Lo extraordinario y ordinario son elementos fundamentales en la construcción de Evangelina, que por un lado es representada a partir de una acción extraordinaria, y, por otro, como una mujer normal y corriente.

Para el análisis del proceso de cambio de Evangelina de ser una persona común a una heroína en pocos días, es útil el concepto de celebrificación, ya que detecta los aspectos que se utilizan para construir la imagen de una estrella (Driessens, 2012, p. 643). Oliva, Pérez-Latorre y Besalú identifican tres rasgos de la celebrificación de candidatos políticos que están vigentes en el caso de Evangelina. El primer rasgo es la representación de la persona como extraordinaria; el segundo, la representación de la persona como normal y corriente, y el tercero, el género en el que aparece el personaje: reality shows, noticias, revistas, etc. Aunque se detectan los tres rasgos de la celebrificación en Evangelina, quiero argumentar que ella no es una celebridad; sin embargo, sí es una heroína. El héroe surge como producto de la demanda de la masa. En otras palabras, hay una percepción nacional de Evangelina en la que la celebridad es popular en el nivel subcultural y su existencia se basa en razones individuales.

En los estudios de heroísmo ordinario, es importante analizar el medio en el que aparecen. En Heroes in a Media Age (1994), Susan Drucker y Robert Cathcart argumentan que los héroes son fenómenos de comunicación; es decir, estudiar a los héroes implica estudiar la comunicación sobre estos personajes. Además, el medio es importante, ya que los diferentes tipos de comunicación construyen distintos tipos de héroes (Wendt, 2016, p. 11). En un estudio sobre la activista peruana María Elena Moyano hecho por Gabriela McEvoy, se demuestra que el discurso periodístico crea una imagen heroica de María Elena Moyano, quien juega el mismo papel tradicional que el héroe ha venido representando dentro de la sociedad desde la antigüedad (McEvoy, 2008). El caso de Evangelina es parecido, de tal manera que el discurso periodístico crea una imagen heroica de ella, quien juega el papel de la madre fuerte que tiene su raíz en las relaciones de género tradicionales de Latinoamérica. 


\section{LA REPRESENTACIÓN SOCIAL DEL GÉNERO FEMENINO EN LOS MEDIOS DE COMUNICACIÓN}

Uno de los temas más desarrollados en relación con Evangelina es el de la mujer. Ser representada como mujer no es una representación neutral y objetiva. La mujer es una construcción cultural que implica ciertos roles, percepciones y conductas de lo femenino y masculino. Es un fenómeno cultural que no es considerado una entidad fija; cambia con el tiempo y con el dinamismo de la sociedad. El significado de qué implica ser hombre o mujer se tiene que entender dentro un contexto social particular (Niklander y Crawford, 2015, p. 1149).

Para el análisis utilizo la revisión de los conceptos de marianismo y machismo planteados por la antropóloga peruana Norma Fuller (1995). Los conceptos expresan los símbolos centrales de la feminidad y masculinidad en países latinoamericanos (p. 1). Según Fuller, el uso de los conceptos no es universal: es, más bien, contextual y corresponde a una lógica jerárquica (p. 14). En la imagen de Evangelina se encuentra la reproducción de estereotipos de género, pero hay que ser conscientes de que es la imagen de un grupo social poco representado en los medios de comunicación. Además, los conceptos son esenciales para el análisis de la relación entre Evangelina y su esposo, y para entender cómo ella es representada como superior a él.

Hay varias investigaciones que afirman que la representación de la mujer en las noticias es sexista, discriminatoria y estereotipada en todo el mundo (Byerly y Ross, 2006; Núñez y Establier, 2008). Los medios de comunicación deben ser una plataforma que aporte diferentes perspectivas, debido a que el lenguaje que utilizan difunde una visión del mundo particular (Niklander, Soto y Crawford, 2015, p. 1149). En este sentido, la imagen de Evangelina construye y (re)produce una idea de la mujer. Según Carolyn Byerly y Karen Ross, quienes analizan la representación de la mujer en los noticieros, esta representación de la mujer se reproduce y normaliza para proteger el statu quo en el cual los hombres son superiores a las mujeres en todo sentido (Byerly y Ross, 2006, p. 54).

En el Perú, ahora se cuestionan en mayor medida temas relacionados con el género, como los feminicidios, el aborto, la sexualización de la mujer, los derechos de las mujeres, entre otros. Esto se ve claramente en el trabajo realizado por $\mathrm{Ni}$ Una Menos en conjunto con la Asociación de Comunicadores Sociales Calandria, que, en las redes sociales, han publicado fotografías de textos como: «Por una comunicación que respeta la dignidad de las mujeres y sus derechos - ¡No más publicidad sexista!» (Concortv.gob.pe, 2016). 
En la «Campaña por una publicidad no sexista» de las organizaciones Flora Tristán, Demus, Calandria, Manuela Ramos y Milena Comunicaciones se problematiza la estereotipación de la mujer en los medios de comunicación. Ellos intentan cambiar el hecho de que «persiste la tendencia a encasillarla en estereotipos: sumisa y delicada, ama de casa, objeto sexual».

Para estudiar la representación de género en el caso de Evangelina, el concepto de ideología es útil. Según Fairclough, ideología es «meaning in the service of power» $(1995$, p. 14). Las ideologías son construcciones de sentido que producen, reproducen o transforman las relaciones de dominación (1992, p. 87). El concepto es útil para estudiar la práctica discursiva como contribución a la creación y reproducción de relaciones de poder desiguales entre grupos sociales (Phillips y Jørgensen, 2002, p. 63), por ejemplo, entre hombres y mujeres. En este sentido, el análisis discursivo sirve para analizar la práctica discursiva y el rol de estas prácticas en los intereses de grupos sociales particulares (Phillips y Jørgensen, 2002).

\section{LA IDENTIDAD SOCIAL DE EVANGELINA}

Para analizar la identidad social de Evangelina he identificado cuatro discursos principales en los periódicos. Con identidad social me refiero a la función de identidad del lenguaje de Fairclough, que tiene que ver con la forma como las identidades son creadas en el discurso (1992, p. 64). Estos discursos son: sobreviviente, mujer, religiosa y pobre. En los artículos se utilizan juntos o separados, pero en este estudio sirven como herramienta de análisis.

\section{Sobreviviente}

A continuación se analiza la representación de Evangelina como sobreviviente, característica que siempre está presente en los artículos. El discurso del sobreviviente es fundamental en la construcción de la heroína ordinaria. Es una característica que identifica a Evangelina como extraordinaria.

Todos los artículos inician identificando a Evangelina como sobreviviente. Algunos ejemplos son los siguientes títulos: «Evangelina, la mujer símbolo de lucha que el huaico no pudo arrasar» $(L R, 15.03 .17)$ y «Mujer que salvó de morir tras ser arrastrada por huaico en Punta Hermosa se recupera satisfactoriamente» (Trome, 16.03.17). Su identidad social está relacionada con el hecho de que sobrevivió al huaico y siempre se describe con palabras que ponen énfasis en 
lo extraordinario. Además, no es representada como una víctima en los títulos, porque tiene agencia. Es el actor activo en las cláusulas «no pudo arrasar» y «se salvó de morir»; son procesos materiales que, según Michael Halliday, son un proceso de hacer en el mundo (Halliday, 1994, p. 207). En este caso, implican dos actores: Evangelina y el huaico, donde Evangelina es el uno que hace en el mundo y el huaico es el beneficiario.

Evangelina Chamorro Díaz (32), la valiente mujer que sobrevivió luego ser arrastrada por un enorme huaico en Punta Hermosa, se ha convertido en un ejemplo de coraje y lucha ante los recientes desastres naturales registrados en todo el país a causa del fenómeno de El Niño $(L R, 16.03 .17)$.

En este ejemplo, el sujeto Evangelina se convierte en una sobreviviente. $L a$ República escribe: «se ha convertido en», que es un proceso existencial. Su identidad cambia de ser común y corriente a ser extraordinaria. El hecho de sobrevivir el huaico la convierte en un ejemplo de coraje.

Al ser representada a partir de una acción extraordinaria y por ser una persona extraordinaria, se convierte en un personaje que merece la atención del mundo. Es una representación que crea una persona admirable que se convierte en un ejemplo a seguir. Además, es una representación que hace posible que salga en los medios de comunicación.

\section{Mujer}

Evangelina es representada como madre desde los primeros artículos que se publicaron sobre ella. Es una característica siempre presente debido a que se la relaciona con su fuerza.

\section{Madre coraje: «Ella tenía dos motivos para vivir: sus dos hijos»}

A partir de la primera entrevista con Evangelina se empieza a representarla como madre y este rol es importante, ya que los periódicos construyen un argumento en el que la maternidad es la fuerza de la mujer. Que Evangelina tenga fuerza es visto como algo extraordinario que tiene que ser explicado. Un ejemplo es el título de La República: «Evangelina Chamorro: 'Pensar en mis hijas me mantuvo con vida'» $(L R, 22.03 .17)$. El tema principal del artículo es la maternidad como elemento clave de la supervivencia de Evangelina. El momento que pasó atrapada dentro el huaico se describe de la siguiente manera: «En el relato, confesó que lo único que la mantuvo con vida fue pensar en sus dos menores hijas. 'Yo 
decía: Señor, dame fuerzas para salir. Yo solo recordaba que mis hijas estaban en el colegio y pensaba en qué será de ellas sin sus padres'» $(L R, 22.03 .17)$. La maternidad es representada como la única razón por cual Evangelina sobrevivió. Según la antropóloga chilena Sonia Montecino, «maternidad y conyugalidad conformarían a las mujeres independientemente de su clase, edad, nacionalidad, etc.» (Montecino, 1996, p. 193). La categoría de la madre es, por lo tanto, una categoría universal que crea una identificación reconocible. Oliva, Pérez-Latorre y Besalú identifican uno de los rasgos en la celebrificación de los candidatos políticos, individuos normales y corrientes, a través de distintos elementos, como son la vida familiar, el estilo de vida y la apertura emocional. La identificación de Evangelina como madre la representa como similar a nosotros, pero al mismo tiempo como un ejemplo a seguir por ser una madre luchadora. Por lo tanto, la celebrificación implica las dos características. «Es importante entender que para que exista celebrificación necesariamente deben darse estos dos polos a la vez» (Oliva, Pérez-Latorre y Besalú, p. 2015).

Evangelina no es identificada como una madre común, más bien se la identifica como un ejemplo a seguir, como indica el subtítulo de La República: «Madre coraje. Sobreviviente de huaico en Punta Hermosa pide ayuda a las autoridades para recuperar su corral de lechones, que fue arrasado por el alud» $(L R, 16.03 .17)$. $\mathrm{Su}$ identidad como madre predomina, aunque la siguiente oración no guarda relación con el hecho de ser madre.

Los periódicos intentan explicar la fortaleza de Evangelina a partir del conocimiento científico. Trome publicó un artículo con el siguiente título «Evangelina Chamorro: ¿qué define a una mujer con fortaleza?» (Trome, 25.03.17). Aquí se describe a Evangelina como una mujer con fortaleza. Posteriormente sigue una explicación psicológica de las mujeres con fuerza. A partir del individuo se describe una característica universal de todas las mujeres con fuerza. La fortaleza de las mujeres es definida a partir de la maternidad, lo cual es evidente en el siguiente subtítulo: «Son aguerridas y luchadoras por sus hijos. Evangelina es un ejemplo» (Trome, 25.03.17). El hecho de ser madre da fortaleza a las mujeres. Otra vez se define a las madres como un grupo homogéneo que, por el hecho de serlo, se sobrentienden aguerridas y luchadoras. Ser un ejemplo significa que las demás mujeres deben ser como Evangelina. Construye un ideal de la mujer con fortaleza y no hay lugar para alternativas, porque la maternidad — es decir, un factor biológico- es la fortaleza. El artículo utiliza a un experto para justificar el argumento: «Pero, ¿cuál es la fuerza que impulsa a una mujer a no dejarse morir y luchar por lo que quiere? Úrsula Calle, psicóloga de la clínica Delgado, 
explica que lo ocurrido con esta valiente pobladora no es un hecho marcado por la suerte» (Trome, 25.03.17). El psicólogo tiene un conocimiento institucionalizado y, por lo tanto, aceptado por la sociedad como verdadero e incuestionable.

El artículo continúa con el punto de vista de la psicóloga: «Señala que la capacidad de soportar cualquier situación difícil se debe a que todas las mujeres poseen el instinto natural de supervivencia y el instinto materno, que les da una ventaja frente a los varones» (Trome, 25.03.17). En esta cita se categoriza a todas las mujeres como iguales por sus instintos naturales. Se apela a la normalidad de Evangelina: ella tiene una característica en común con todas las mujeres. A la misma vez, la maternidad es naturalizada como la fortaleza de la mujer y la única manera de ser superior al hombre, porque él no puede tener hijos (dar a luz). Según esta lógica, una mujer fuerte necesariamente tiene que ser madre.

La fuerza de Evangelina para sobrevivir es tratada como algo extraordinario. Es decir, la fuerza de una mujer no es algo esperable; por lo tanto, tiene que ser explicada y analizada, además, por una psicóloga. En una lógica así, la fuerza depende del género y no de la persona.

\section{El hombre de su vida y la madre de sus hijos: las relaciones de género}

Evangelina es representada en relación con su esposo, Armando, quien también fue llevado por el huaico. En el texto del artículo de Trome se observa que la elección de palabras para representar a los dos es muy diferente: «Evangelina Chamorro volvió a la vida y hoy pudo volver a abrazar al hombre de su vida, aquel que nunca la dejó sola y lloraba al narrar el momento en que el huaico de Punta Hermosa estuvo a punto de arrebatarle la vida de la madre de sus hijos» (Trome, 22.03.17). Por un lado, «madre de sus hijos» la representa a partir de la relación con los hijos; por otro, «el hombre de su vida» sostiene la sexualidad de la mujer a un hombre, su esposo. Ambos periódicos representan las relaciones de familia en las que la mujer es madre, y el hombre, esposo. Según Wendt, los héroes (hasta el inicio del siglo XX) justifican relaciones de género tradicionales (2016, p. 13). Por un lado, el hombre es relacionado con la esfera pública y «sus características son responsabilidad y protección hacia adentro y preeminencia y virilidad hacia afuera» (Fuller, 1995, p. 4). En cambio, la mujer es «la reina del hogar» y «la encarnación de los valores asociados a la intimidad, el afecto y la lealtad de grupo» (Fuller, p. 1). Esto se observa, ya que a Evangelina se le relaciona con la crianza de los hijos; es decir, ella es el sustento de la familia en la que el hombre es el protector. En la fotografía (1), que acompaña el artículo, el esposo y Evangelina están abrazándose. Ella vestida en 


\section{Fotografía 1. Evangelina y su esposo abrazándose}

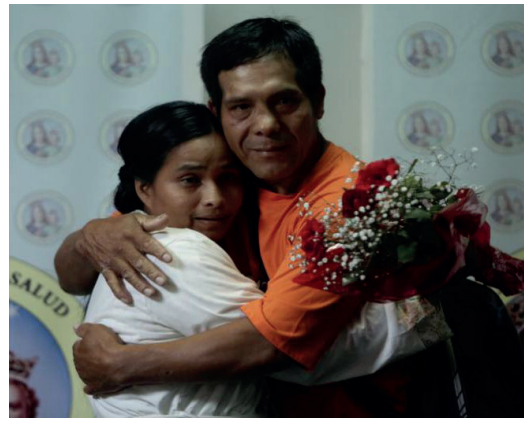

Fuente: Trome, 22.03.17.

ropa de paciente y él en ropa de calle. Estas características en la forma de vestir de ambos construyen una imagen de Evangelina como la sensible y él como su protector. Evangelina es la protectora de sus hijos, pero el esposo es representado como el protector de ella. Él la protege, la abraza y le da rosas rojas, un símbolo de la maternidad y fragilidad de la mujer. La representación de género no ocurre unívocamente. Según Fuller, hay que ser consciente de que las sociedades latinoamericanas experimentan un proceso de modernización que se cuestiona la jerarquía tradicional (1993, p. 4). El esposo llora cuando habla del riesgo de perder a Evangelina, la abraza, se queda muchas veces en el fondo y no habla por ella; es decir, Evangelina domina en el espacio público y él se queda en el fondo, porque su presencia solo es requerida en relación con Evangelina. La supervivencia de ella se debe a los hijos; entonces, el hecho de ser madre - una característica femenina que no cuestiona la jerarquía tradicional de género - la hace acceder a la esfera pública y dominarla.

\section{Religiosa}

A continuación se analiza, en primera instancia, cómo se relaciona a Evangelina con la virgen María, y en segunda instancia, cómo se representa a Evangelina como creyente.

\section{Mujer y madre religiosa}

Los artículos de Trome y La República que tratan sobre la primera entrevista que se hizo a Evangelina adjuntan una fotografía (2) en la que se observa a Evangelina sentada frente la imagen de la virgen María Auxiliadora, que también es el símbolo del Ministerio de Salud. En la fotografía se puede observar a Evangelina mirando 
Fotografía 2. Evangelina dando la primera entrevista en el hospital

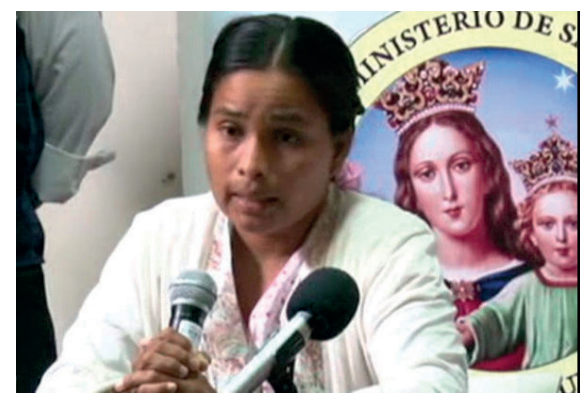

Fuente: La República, 22.03.17.

hacia abajo y a la virgen mirando hacia Evangelina. La virgen está cargando al niño Jesús y ambos llevan coronas. La virgen representa la maternidad absoluta, la mujer abocada a su destino reproductivo y a la protección del hijo. La mirada de la virgen vigila y protege a Evangelina. Parece como si el niño Jesús tocara la espalda de Evangelina y le diera su bendición. Evangelina tiene en su mano un micrófono. Ella está hablando con el público, es decir, ella tiene voz. Es la mediadora entre lo sagrado - la virgen y el niño Jesús- y el hombre común. Fuller propone que «la mujer es también mediadora entre lo sagrado y profano, entre grupos políticos y clases sociales (la virgen actúa como intermediaria entre Dios y los hombres), las madres practican la caridad entre los pobres de manera de suavizar los conflictos entre la parentela y entre las clases sociales» (Fuller, 1995, p. 7). En esta fotografía, Evangelina se presenta como extraordinaria, porque conecta lo humano con lo espiritual. Lo importante no es su identidad relacionada a su clase social ni raza sino más bien, como madre. También se puede observar en el título del artículo: «Pensar en mis hijos me mantuvo con vida» $(L R, 22.03 .17)$. Para las madres católicas proteger a los hijos es el sentido de la vida. Por lo tanto, este une a todos sin importar su clase social, etnicidad, etc.

Esta lógica, donde la fuerza de la mujer se obtiene del hecho de ser madre, la ubica dentro la tradición del catolicismo en la cual la imagen de la virgen representa la humildad y el sacrificio infinito de la mujer frente al hombre, que es inferior moralmente (Fuller, 1995, p. 2). Evangelina representa la imagen católica de la mujer: «La madre y la virgen se asocian a pureza sexual y a 'la casa'» (Fuller, p. 8), es la mujer que no es un peligro para los hombres, padres y hermanos, cuyo papel es de guardianes y protectores de la mujer. La mujer es relacionada con la casa y «la mujer que vive su sexualidad es asimilada simbólicamente al 
desorden y al peligro, es decir, a 'la calle'» (Fuller, p. 9). Por lo tanto, la imagen de Evangelina representa los valores tradicionales religiosos del género, no los cuestiona y los representa como mediadora. Eso significa que ella es un sujeto que tiene acceso al público y puede opinar.

El discurso religioso crea una heroína que representa los valores tradicionales del género relacionados con la religión. Es decir, la imagen de Evangelina tiene una relación fuerte con la imagen de la virgen; sin embargo, no es representada en relación a una institución religiosa, lo que se analiza y detalla a continuación.

\section{«Gracias a Dios»}

La religión es un elemento interesante, ya que ningún periódico menciona que Evangelina es evangélica y no católica. Al no mencionarlo en los artículos, consiguen una mayor identificación con Evangelina y el enfoque en la relación con Dios como una entidad fuera de las religiones. Esto se observa en las siguientes citas donde Evangelina cuenta cómo pedía ayuda de Dios cuando estaba en el lodo: «'Llegué al puente de Punta Hermosa y en ese momento le pedí ayuda a Dios y es gracias a Dios que me diera fuerza', agregó Evangelina Chamorro» (Trome, 22.03.17). Mediante el agradecimiento a Dios, Evangelina justifica su supervivencia no como fruto de la lucha personal, sino como producto de una ayuda divina. «El significado originario de esta locución es explotado para reducir o para minimizar la responsabilidad personal en el éxito conseguido» (Meléndez, 2003, p. 103). El éxito de Evangelina es fruto de una ayuda divina. En este sentido, el éxito está posicionado fuera de ella. Esta posición ubica la responsabilidad del éxito en las manos de Dios y no en el individuo. Además, Dios es una entidad fuera la religión; es decir, la Iglesia y la comunidad religiosa no están presentes en los relatos, es más bien, un individualismo religioso el que domina.

Según Ian Watt, este es un rasgo del mito del individualismo moderno. Su análisis del cuento de Robinson Crusoe resalta una relación entre el individuo y la religión, pero una relación en la que no hay comunidad de creyentes: «Al contrario que en el catolicismo, no hay misa, no hay confesión» (Watt, 2003, p. 175). Igual que en el caso de Evangelina, la relación de la persona con Dios es lo que la salva y esta relación no involucra a la comunidad. Evangelina no está relacionada con una comunidad religiosa: es más bien el individuo y su relación con Dios lo que la salva de la imponente fuerza de la naturaleza. Una representación así no permite distinguir religiones. Constituye una norma de la sociedad en la que el verdadero Dios es el Dios católico; por lo tanto, domina los valores católicos, donde por ejemplo la virgen juega un papel importante. 


\section{Pobre}

Esta categoría no es la más visible, aunque es un elemento siempre presente. Evangelina vive en un terreno ilegal, emigrante de la selva, criadora de animales y maneja una mototaxi. Debido a esto, ella no es representada como una pobre extraordinaria: ella se diferencia del resto.

\section{MUJER DE HIERRO}

Un ejemplo de la construcción de Evangelina como extraordinaria son las palabras que se utilizan para hablar sobre ella. Cuando Evangelina es trasladada al hospital público María Auxiliadora, la ministra de Salud, Patricia García, la visita y ella es la primera persona en brindar declaraciones a los medios de comunicación sobre el estado de salud de Evangelina. Una de las expresiones que se utiliza para describir a Evangelina en un artículo de Trome es «Mujer de Hierro». El texto es: «Los médicos que atendieron a la denominada 'Mujer de Hierro' informaron a la ministra de Salud que ella solo sufrió arañones y golpes pero que en general su estado de salud es muy bueno — pues además está consciente-, y que se recuperará satisfactoriamente» (Trome, 16.03.17). La expresión mencionada surge del periodista, quien muestra afinidad con el nombre. No indica quién la ha denominado «Mujer de Hierro», pero como la fuente es la ministra de Salud, se asume que ella es la autora. La Mujer de Hierro — con mayúsculas, lo que indica que es un nombre - es una metáfora que utilizamos para construir una realidad particular.

Mujer de Hierro tiene un correlato en el inglés «Iron Lady», que se refiere a una mujer con mucha influencia política. Por ejemplo, se utiliza para designar a Margaret Thatcher, como se nota en la biografía de John Campbell Margaret Thatcher Volume Two: The Iron Lady (2003). La palabra lady traducida al español es dama, pero la ministra de Salud utiliza la palabra mujer. Lady refiere a una dama de estatus social alto, que tiene poder y autoridad, como es el caso de Margaret Thatcher. Al utilizar la palabra mujer se alude a un sujeto que no es de clase alta, pero el hierro funciona como una metáfora que representa algo fuerte y determinado. La metáfora representa a Evangelina como una mujer extraordinaria de la clase social baja. Una construcción así no significa una amenaza a la identidad política de la ministra y la clase alta: es, más bien, un contraste a ellas. Si Evangelina fuera una dama, tendría influencia política y autoridad, pero al llamarla mujer, no se cuestionan las desigualdades de clase ni las autoridades, más 
bien, se establecen las normas de la sociedad. Se construye una mujer extraordinaria dentro una clase social particular a la cual la ministra no pertenece. Trome es un periódico cuya audiencia objetiva es justamente la clase baja y media. Al llamar a Evangelina mujer y no dama, se crea una identificación con la clase baja que es la audiencia objetiva. Además, la categoría mujer es más inclusiva que la categoría dama, porque incluye a todas las mujeres, mientras que dama se relaciona con una clase social.

\section{¿QUÉ SIGNIFICA SER MEJOR?}

Otro artículo que pone énfasis en lo extraordinario de Evangelina es uno publicado por el Día de la Madre en La República. El título del artículo es: «Evangelina Chamorro: 'Quisiera que mis dos niños sean mejores que yo. Por ellos, doy todo'» $(L R, 13.05 .17)$. En el artículo, el control de interacción sigue un modelo entrevistador y entrevistado donde el periodista hace preguntas y Evangelina contesta. Después de una presentación corta de Evangelina, se comienza con las preguntas. Las primeras son: «¿cómo se encuentra ahora?», «¿sigue trabajando?», «¿sigue criando ganado o se dedica ahora a otras actividades?», «se viene el Día de la Madre y usted es mamá de dos pequeñas», y después hay más preguntas sobre la maternidad. Las preguntas introducen nuevos temas en el siguiente orden: salud, trabajo y maternidad. La maternidad es el tema principal del artículo, pero es el último tema que se desarrolla, porque se relaciona con el trabajo y la clase social. Ella es descrita como trabajadora, lo que puede referirse a una persona que tiene cualquier tipo de trabajo, pero también puede referirse a una clase social.

El trabajo ubica a Evangelina dentro de cierta clase social. Hay entonces un intento por ubicar a Evangelina dentro de una clase social. Esto posiblemente se deba a que los lectores de La República son en su mayoría de una clase socioeconómica más alta que la de Evangelina. En lugar de universalizar a las mujeres como iguales, como en el artículo de Trome sobre la mujer con fuerza, aquí se identifica a Evangelina como una mujer de clase social baja. Ella es representada como madre fuerte por el hecho de querer crecer económicamente y por trabajar al igual que su esposo.

En la introducción del artículo se dice: «Como madre luchadora y trabajadora, ella y su esposo son el sustento de un nuevo hogar». La palabra que la describe es trabajadora y no ama de casa, como en otros artículos. Hay una división igualitaria entre los géneros en lo respectivo al trabajo. Al preguntar a Evangelina sobre el trabajo, se generan preguntas que indirectamente se 
cuestionan por la división de trabajo entre ella y su esposo. Evangelina y su esposo trabajan como criadores y tenían su corral junto a su casa. Cuando le preguntan: «¿sigue criando ganado o se dedica ahora a otras actividades?», una parte de su respuesta es: «mi esposo y yo trabajamos juntos» y «como mujer y hombre, nos repartimos las tareas de la casa, a veces en el corral, otras veces en mi casa». En este sentido, la esfera privada y la esfera pública (trabajo) son compartidas por ambos. Evangelina como madre no solo es representada en relación con sus hijos, sino también por el hecho de trabajar de igual manera que el hombre y viceversa. La relación entre género y clase social es sostenida en la fotografía (3) que acompaña el artículo. Evangelina está sentada dentro de un mototaxi en el asiento del chofer, con una mano en el volante. El mototaxi es un medio que se utiliza para transportar personas y es muy barato. Según Pau Avellaneda, este medio de transporte es relacionado con la pobreza en Lima (Avellaneda, 2008). Muchas personas con recursos bajos se ven obligadas a vivir lejos de sus posibilidades de empleo; por lo tanto, están en la necesidad de utilizar este medio de transporte de bajo costo (p. 17). El mototaxi es un instrumento que va a permitir a una persona trabajar y ganar dinero para mantener a su familia. En este caso, la madre está asociada con el trabajo y, por lo tanto, con la esfera pública, aunque la mayoría de los mototaxistas son hombres y el hecho de conducir se asocia con lo masculino. Cabe resaltar que la empresa Crosland utiliza la imagen de Evangelina para vender su producto a una clase social representada por ella. $\mathrm{Su}$ imagen como cara del producto genera una identificación con Evangelina. Su audiencia objetiva es, por lo tanto, madres emprendedoras.

\section{Fotografía 3. Evangelina sentada dentro un mototaxi}

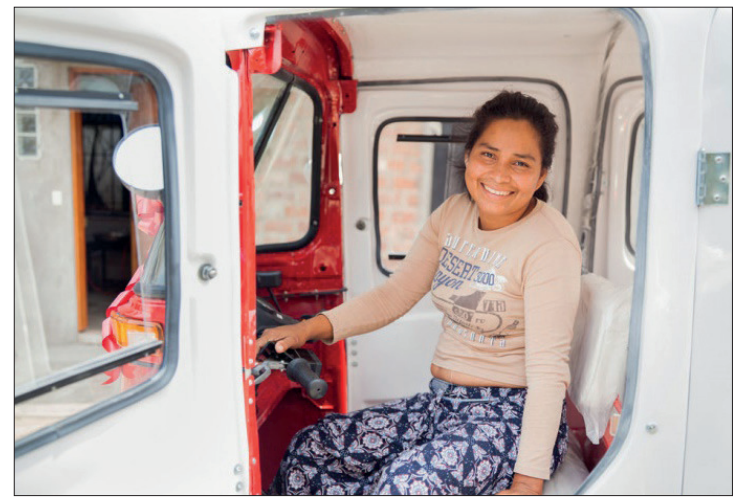

Fuente: La República, 13.05.17. 
Evangelina no solo es representada como una persona de bajos recursos económicos. En su imagen también se ve a la madre emprendedora que tanto aparece en los medios de comunicación en los últimos años. Evangelina es la madre emprendedora que hace su negocio desde casa; es decir, puede cuidar sus hijos, hacer las tareas domésticas y ganar dinero al mismo tiempo. Se configura como el sujeto moderno ideal para el mercado libre (Godoy, Rodríguez, Santos, Tapia, Villablanca, Villarreal y Zúñiga, 2018, p. 323). Ella es el sujeto que mediante su esfuerzo personal aporta al desarrollo humano con su negocio de crianza de «sus animalitos». Evangelina es la madre moderna que es también esposa y profesional. Tres modelos que conviven y entran en conflicto se superponen. Fuller señala que la maternidad es el punto focal de la identidad de la mujer latina y que le da el acceso al poder doméstico, el estatus sagrado y el reconocimiento de sus hijos (1993, p. 38). Ser madre es la identidad que predomina en la representación de Evangelina, pero no la excluye de ser emprendedora.

En cuanto al título, «Quisiera que mis dos niños sean mejores que yo» ( $L R$, 13.05.17), ser mejor puede tener diferentes significados. La palabra «mejor» está vinculada con el primer tema tratado: el trabajo, que a su vez está relacionado con cierta clase social. Mejor se refiere al trabajo; es decir, que sus hijos tengan mejores oportunidades laborales de las que tuvo ella para que puedan crecer económicamente. Ser madre luchadora implica trabajar fuerte para que sus hijos tengan mejores posibilidades económicas. Según Ian Watt, el Homo economicus en el relato de Robinson Crusoe «pone de manifiesto que un hombre normal y corriente, a solas, es capaz de someterse a la naturaleza y sojuzgarla a sus propios intereses materiales, y que a la sazón triunfa frente al entorno físico» (Watt, 2003, p. 164). Evangelina está representada a partir del mismo individualismo económico. Ella es capaz de someterse a la naturaleza y ahora a la preocupación de crecer económicamente. Como indica la introducción del artículo:

Nada trae abajo el espíritu de una mujer luchadora. A casi dos meses del huaico que le hizo perder todo, menos la fe, Evangelina sigue de pie, con más fuerzas que nunca. Como madre luchadora y trabajadora, ella y su esposo son el sustento de su nuevo hogar. Aquí nos cuenta más acerca de su principal motivación: sus dos pequeñas hijas $(L R, 13.05 .17)$.

En esta cita, la fuerza de la madre es la fuerza de crecimiento económico; es decir, recuperar el trabajo para sustentar su nueva casa y el motivo para hacerlo son los hijos. Es una lógica plenamente económica, en la que la madre luchadora 
es aquella que trabaja fuerte para que sus hijos crezcan económicamente y, por lo tanto, «asciendan» de clase social. Es un intento de salir de su clase social y entrar en otra: las hijas quieren ser policía y doctora.

\section{CONCLUSIONES}

El análisis crítico del discurso de la representación de Evangelina en los medios de comunicación es mayoritariamente una herramienta para analizar los discursos que representan a Evangelina en los medios. Se han podido analizar los cuatro discursos descritos anteriormente: sobreviviente, mujer, religiosa y pobre. Estos discursos son difíciles de separar, porque se entrecruzan y uno influye en el otro, pero analizarlos ha servido para entender, con toda amplitud, la identidad social de Evangelina.

El análisis muestra que Evangelina está principalmente representada como sobreviviente. Un sobreviviente es creado a partir de lo extraordinario, por ejemplo, mediante ciertas palabras utilizadas para describir su identidad y dándole agencia. Además, el elemento de lo extraordinario es una característica presente en todos los discursos analizados: es una madre extraordinaria, una religiosa extraordinaria y una pobre extraordinaria.

Ser una mujer fuerte es una construcción que necesita explicación. Trome utiliza un discurso científico cuyo argumento es que un factor biológico, la maternidad, es la fuerza de la mujer y también el medio para superar al hombre. La maternidad es, entonces, una característica que representa a la maternidad como la fuerza de todas mujeres, fuera de factores como clase social, etnicidad, sexualidad, etc. Una representación así homogeneiza a las mujeres, a la misma vez que tiene la función de unirlas.

Representar a Evangelina como religiosa ha sido otro elemento importante. Evangelina es la madre que se sacrifica por la familia, al igual que la virgen María. Evangelina representa los valores de la virgen y establece las normas de la sociedad en las que el deber de la mujer es tanto la familia como la casa. Se representa a Evangelina como mediadora entre lo sagrado y el público, lo que le da el poder de acceder al discurso público. Es decir, tiene voz y puede expresar su opinión.

Evangelina es representada como la pobre extraordinaria porque ella y su esposo son iguales en el trabajo y en la casa y porque se sacrifica por sus hijos para darles mejores oportunidades. Evangelina es madre y emprendedora, dos identidades que conviven, una representación que no cuestiona la identidad principal de la mujer que es ser madre, pero sí la modifica. Se puede observar 
que la madre no es una identidad aislada: es una identidad compleja que cambia y depende del contexto.

Evangelina ha llegado a representar un grupo social que no es muy representado en los medios de comunicación. Ella resulta ser una persona que vuelve a aparecer en los medios de comunicación y que sigue siendo representada como el ejemplo de una mujer fuerte. Ella tiene, por lo tanto, un significado político, dado que tiene voz y poder de influencia dentro de la sociedad. En el año 2018, ella aún sigue apareciendo en la prensa y sigue siendo un tema mediático que no pasa de moda. Por ejemplo, es celebrada por la municipalidad de Lima en el Día de la Mujer, así como catalogada como una de las personas más importantes en el Perú del año 2017. Resalto que hay que ser conscientes del uso de Evangelina, porque es un uso político en el que se celebra el comportamiento de una mujer que representa valores tradicionales y dominantes en la sociedad. El análisis muestra que los discursos que se utilizan para representar a Evangelina reproducen los valores tradicionales de género y mantienen las estructuras sociales dominantes, pero también las modifican y cambian según el contexto.

\section{REFERENCIAS BIBLIOGRÁFICAS}

Avellaneda, Pau (2008). Movilidad cotidiana, pobreza y exclusión social en la ciudad de Lima. Anales de Geografía, 28(2), 9-35.

Byerly, Carolyn M. y Karen Ross (2006). Woman and Media. A Critical Introduction. Malden, MA: Blackwell Publishing.

Campbell, John (2003). Margaret Thatcher : Volume 2 the Iron Lady. Londres: Jonathan Cape.

Compañía Peruana de Estudios de Mercados y Opinión Pública S.A.C (2017). Estudio de lectoría de diarios en Lima y 15 principales ciudades 2016. Recuperado de http://cpi.pe/images/upload/paginaweb/archivo/23/LectoriaDiarios_2016.pdf

Driessens, Olivier (2012). The celebritization of society and culture: Understanding the structural dynamics of celebrity culture. International Journal of Cultural Studies, 16(6), 641-657. https://doi.org/10.1177/1367877912459140

Fairclough, Norman (1992). Discourse and Social Change. Cambridge: Polity Press.

Fairclough, Norman (1995). Media Discourse. Londres: Edward Arnold.

Flora Tristán (s.f.). Campaña contra la publicidad sexista. Recuperado el 22.06 .18 de http://www.flora.org.pe/web2/index.php?option=com_content\&view=article\&i $\mathrm{d}=214 \&$ Itemid $=91$ 
Fuller, Norma (1993). Dilemas de la femineidad: mujeres de clase media en el Perú. Lima: Fondo Editorial de la Pontificia Universidad Católica del Perú.

Fuller, Norma (1995) Acerca de la polaridad machismo y marianismo. En Luz Gabriela Arango, Magdalena León, Mara Viveros (eds.), Lo femenino y lo masculino: estudios sociales sobre las identidades de género en América Latina (pp. 241264). Bogotá: Third World Editions, Ediciones UniAndes.

Godoy, Carmen Gloria, Manuela Rodríguez, Angélica Santos, Nicole Tapia, Gittel Villablanca, Gabriela Villarreal, Soledad Zúñiga (2018). Género y emprendimiento. Análisis crítico en torno a la subjetividad de emprendedores y emprendedoras de la Región Metropolitana, Chile. Revista de Antropología Experimental, 18, 231-247.

Halliday, M. A. K (1994). An Introduction to Functional Grammar. 3r ${ }^{\mathrm{d}}$ Edition. Londres: Edward Arnold.

McEvoy, Gabriela (2008). La construcción de la imagen heroica a través del discurso periodístico. El caso de la activista peruana María Elena Moyano. Historia Crítica, 35, 82-104. https://doi.org/10.7440/histcrit35.2008.06

Meléndez, Carlos (2013). Hacia un modelo de caracterización lingüística de las locuciones evacuativas del español actual: el caso de «gracias a Dios». Lingüística Española Actual, 35(1), 87-108.

Montecino, Sonia (1996). Identidades de género en América Latina: mestizajes sacrificios y simultaneidades. En Luz Gabriela Arango, Magdalena León y Mara Viveros (comps.), Género e identidad. Ensayos sobre lo femenino y lo masculino. Bogotá: Uniandes, Facultad de Ciencias Humanas, 1997.

Muñoz, Fanni G. (2016). Discursos sobre el feminicidio en la prensa escrita: El Comercio (2012) y Trome (2013): entre la visibilización e invisibilización del fenómeno. En Feminismos y poscolonialidad, 2 (pp. 119-151). Buenos Aires: EGodot.

Niklander, Stefanie, Ricardo Soto y Broderick Crawford (2015). Representaciones del género femenino en medios de comunicación digitales vía análisis crítico del discurso: un caso de estudio. Female Gender Representations in Digital Mass Media via Discourse Analysis: A Case Study. CISTI (Iberian Conference on Information Systems \& Technologies / Conferência Ibérica de Sistemas e Tecnologias de Informação) Proceedings, 1, 1147-1152.

Núñez, Sonia y Helena Establier (2008). La representación/presencia de la mujer en los medios de comunicación. Feminismo/s, 11.

Oliva, Mercé, Óliver Pérez-Latorre y Reinald Besalú (2015). Celebrificación del candidato. Cultura de la fama, marketing electoral y construcción de la imagen pública del político. Arbor, 191(775), a270. https://doi.org/10.3989/arbor.2015.775n5009

Phillips, Louise y Louise Jørgensen (2002). Discourse Analysis as Theory and Method. Londres: Sage. https://doi.org/10.4135/9781412983921 
Povedák, István (2014). From Heroes to Celebrities. Problems of definition and potential solutions. En István Povedák (ed.), Heroes and Celebrities in Central and Eastern Europe (pp. 7-17). Szeged, Hungría: MTA-SZTE Vallási Kultúrakutató Csoport.

Watt, Ian (2003). Mitos del individualismo moderno. Madrid: Akal.

Wendt, Simon (2016). Extraordinary ordinariness - Everyday heroism in the United States, Germany, and Britain, 1800-2015. Fráncfort: Campus Verlag.

\section{Fuentes analizadas}

Trome

Redacción Trome. «Evangelina Chamorro y su doloroso testimonio tras sobrevivir al huaico de Punta Hermosa». Trome, 22.03.17. https://trome.pe. Acceso: 15.01.18.

Redacción Trome: «Mujer que salvó de morir tras ser arrastrada por huaico en Punta Hermosa se recupera satisfactoriamente». 16.03.17. https://rome.pe. Acceso: 16.01.18. http://trome.pe/actualidad/punta-hermosa-mujer-arrastradahuaico-43307

Redacción Trome. "Qué define a una mujer con fortaleza». Trome, 25.03.17. https:// trome.pe. Acceso: 15.01.18.

\section{La República}

(Autor desconocido). «Evangelina Chamorro: 'Pensar en mis hijas me mantuvo con vida'». La República, 22.03.17. https://larepublica.pe. Acceso: 15.01.18.

(Autor desconocido): «Evangelina, la mujer símbolo de lucha que el huaico no pudo arrasar». La República, 15.03.17. https://larepublica.pe. Acceso: 10.03.19.

Bazán, Sigrid. «Evangelina Chamorro: 'Quisiera que mis dos niños sean mejores que yo. Por ellas, doy todo'». La República, 13.05.17. https://larepublica.pe. Acceso: 15.01.18.

Gamboa, Edgar. «Evangelina Chamorro: lo perdió todo por huaico, pero se recupera en hospital». La República, 16.03.2017. https://larepublica.pe. Acceso: 15.01.18.

\section{Páginas web}

Concor Tv (Consejo Consultivo de Radio y Televisión)

Concor Tv: «\#NiUnaMenos: También exigen respeto hacia la mujer en los medios de comunicación»16.08.16. https://concortv.gob.pe. Acceso: 20.06.18. 